PROCEEDINGS OF THE

AMERICAN MATHEMATICAL SOCIETY

Volume 136, Number 11, November 2008, Pages 3831-3838

S 0002-9939(08)09364-7

Article electronically published on June 2, 2008

\title{
SIGN CHANGES OF HECKE EIGENVALUES OF SIEGEL CUSP FORMS OF DEGREE 2
}

\author{
AMEYA PITALE AND RALF SCHMIDT \\ (Communicated by Ken Ono)
}

\begin{abstract}
Let $\mu(n), n>0$, be the sequence of Hecke eigenvalues of a cuspidal Siegel eigenform $F$ of degree 2. It is proved that if $F$ is not in the Maaß space, then there exist infinitely many primes $p$ for which the sequence $\mu\left(p^{r}\right), r>0$, has infinitely many sign changes.
\end{abstract}

\section{INTRODUCTION AND PRELIMINARIES}

Let $F \in S_{k}(\mathrm{Sp}(4, \mathbb{Z}))$ be a cuspidal Hecke eigenform of degree 2. Let $\mu(n)$, $n>0$, be the Hecke eigenvalues. It is known that $F$ is in the Maaß space (i.e., $F$ is a Saito-Kurokawa lifting) if and only if $\mu(n)>0$ for all $n$; see [2]. On the other hand, Kohnen has recently proved (see [6]) that if $F$ is not in the Maaß space, then the sequence $\mu(n), n>0$, has infinitely many sign changes. The proof made use of the (as yet unpublished) Ramanujan estimate for non-Saito-Kurokawa cusp forms.

In this note we will strengthen the result on sign changes as follows: If $F$ is not in the Maaß space, then there exist infinitely many primes $p$ for which the sequence $\mu\left(p^{r}\right), r>0$, has infinitely many sign changes. Our proof is also based on a Ramanujan type result for the underlying group $\mathrm{GSp}_{4}$, however not the full Ramanujan conjecture. We use the weaker statement, proved in 4], that a suitable product of Satake $p$-parameters has absolute value one. We showed in [9] that this result, combined with the classification of unitary, spherical representations of $\mathrm{GSp}_{4}\left(\mathbb{Q}_{p}\right)$, implies certain restrictions on the local components of the cuspidal, automorphic representations of $\mathrm{GSp}_{4}(\mathbb{A})$ attached to classical eigen-cusp forms. It is these restrictions on the local representations that are exploited in the present paper.

In fact, our proof works for cusp forms with level as well (as long as the weight is greater than 2). In this case the statement " $F$ is not in the Maaß space" is to be replaced by " $\pi_{F}$ is not a theta lifing from the metaplectic group $\widetilde{\mathrm{SL}}_{2}(\mathbb{A})$ "; here, $\pi_{F}$ is one of the cuspidal, automorphic representations of $\mathrm{GSp}_{4}(\mathbb{A})$ attached to $F$. For the full modular group these two statements are equivalent, and are equivalent to the fact that $\pi_{F}$ is a CAP representation with respect to the Siegel parabolic subgroup. For a higher level it is at least conceivable that $\pi_{F}$ might be CAP without being a theta lifting (namely, $\pi_{F}$ might be a non-trivial twist of a

Received by the editors May 15, 2007, and, in revised form, October 2, 2007.

2000 Mathematics Subject Classification. Primary 11F46.

(C)2008 American Mathematical Society Reverts to public domain 28 years from publication 
theta lifting). Hence, for modular forms with level, we prefer not to translate the "not a theta lifting" condition into purely classical language.

We will now recall the required results from our paper [9]. Let $F \in S_{k}\left(\Gamma_{0}(N)\right)$ be a Siegel cusp form of weight $k>2$ and level $N \geq 1$. Assume that $F$ is a Hecke eigenform with eigenvalues $\mu(n)$ for all $n$ coprime to $N$. As explained in 9, section $2, F$ generates a space $V_{F}$ of cuspidal automorphic forms on $\mathrm{GSp}_{4}(\mathbb{A})$ invariant under right translation. This space may not be irreducible, but does decompose into a finite number of irreducible, cuspidal, automorphic representations. Let $\pi_{F}$ be one of these irreducible pieces. We can write $\pi_{F}$ as a restricted tensor product $\bigotimes \pi_{F, p}$, where $\pi_{F, p}$ is an irreducible, admissible representation of $\operatorname{GSp}_{4}\left(\mathbb{Q}_{p}\right)$. For a prime $p \nmid N$, the equivalence class of $\pi_{F, p}$ is independent of the choice of the irreducible component $\pi_{F}$ of $V_{F}$. For such primes, $\pi_{F, p}$ is the unramified (spherical) constituent of a representation $\chi_{1} \times \chi_{2} \rtimes \sigma$ induced from the character

$$
\left(\begin{array}{cccc}
a_{1} & * & * & * \\
0 & a_{2} & * & * \\
0 & 0 & \lambda a_{1}^{-1} & 0 \\
0 & 0 & * & \lambda a_{2}^{-1}
\end{array}\right) \mapsto \chi_{1}\left(a_{1}\right) \chi_{2}\left(a_{2}\right) \sigma(\lambda)
$$

of the Borel subgroup; here, $\chi_{1}, \chi_{2}, \sigma$ are unramified characters of $\mathbb{Q}_{p}^{\times}$. Since $\pi_{F, p}$ has trivial central character, we have $\chi_{1} \chi_{2} \sigma^{2}=1$. We set $a:=\sigma(p)$ and $b:=\sigma(p) \chi_{1}(p)$.

From [1, Proposition 3.35, we get the relation between Hecke eigenvalues $\mu\left(p^{r}\right)$ for $r>0$ and $a$ and $b$ from the following formal power series identity:

$$
\sum_{r=0}^{\infty} \frac{\mu\left(p^{r}\right)}{\left(p^{r}\right)^{k-\frac{3}{2}}} X^{r}=\frac{1-p^{-1} X^{2}}{(1-a X)\left(1-a^{-1} X\right)(1-b X)\left(1-b^{-1} X\right)} .
$$

Using partial fractions and geometric series, we have shown in [9, Proposition 4.1, that

$$
\frac{\mu\left(p^{r}\right)}{\left(p^{r}\right)^{(k-3 / 2)}}=A_{a, b}(r)+(1-1 / p) \sum_{t=1}^{[r / 2]} A_{a, b}(r-2 t),
$$

where

$$
A_{a, b}(j)=\left(\sum_{u=0}^{j} a^{j-u} b^{u}\right)\left(\sum_{u=0}^{j}(a b)^{-u}\right) .
$$

In [9], Theorem 3.2, we have shown that only three types of local representations of $\pi_{F, p}$ can occur:

1.1 Proposition. Let $N$ and $k$ be positive integers with $k>2$. Let $F \in S_{k}\left(\Gamma_{0}(N)\right)$ be a Hecke eigenform. For $p \nmid N$ let $\pi_{F, p}$ be the corresponding local representation of $\mathrm{GSp}_{4}\left(\mathbb{Q}_{p}\right)$. Then $\pi_{F, p}$ can only be one of the following:

(T) $\chi_{1} \times \chi_{2} \rtimes \sigma$ irreducible with $\left|\chi_{1}\right|=\left|\chi_{2}\right|=|\sigma|=1$ (the tempered case); or

(C) $\chi_{1} \times \chi_{2} \rtimes \sigma$ irreducible with $\chi_{1}=\nu^{\beta} \chi, \chi_{2}=\nu^{\beta} \chi^{-1},|\chi|=1, e(\sigma)=-\beta$ with $0<\beta<1 / 2$ (the complementary series case); or

(SK) $\chi \mathbf{1}_{\mathrm{GL}(2)} \rtimes \sigma$, the spherical constituent of $\nu^{1 / 2} \chi \times \nu^{-1 / 2} \chi \rtimes \sigma$, with $|\chi|=1$ (the Saito-Kurokawa case). 
The characters $\chi_{1}, \chi_{2}, \chi$ and $\sigma$ above are unramified, and $\nu$ is the $p$-adic valuation of $\mathbb{Q}_{p}$ normalized such that $\nu(p)=p^{-1}$.

\section{LOCAL RESULTS}

We now want to give a result on the signs of the Hecke eigenvalues $\mu\left(p^{r}\right)$ for some sub-family of local representations.

2.1 Lemma. Let $F \in S_{k}\left(\Gamma_{0}(N)\right), k>2$, be a Hecke eigenform of degree 2 with eigenvalues $\mu(n)$ for $(n, N)=1$. For $p \nmid N$ let $\pi_{F, p}$ be the corresponding local representation; it is the spherical constituent of an induced representation $\chi_{1} \times \chi_{2} \rtimes \sigma$ as above. Let $a=\sigma(p)$ and $b=\sigma(p) \chi_{1}(p)$. If at least one of $a$ and $b$ is real, then we have exactly one of the two following situations:

1. $\mu\left(p^{r}\right) \geq 0$ for all $r>0$,

2. $\mu\left(p^{r}\right)>0$ for $r \equiv 0(\bmod 2)$ and $\mu\left(p^{r}\right)<0$ for $r \equiv 1(\bmod 2)$.

Proof. The condition on the values $a$ and $b$ implies that $\pi_{F, p}$ is either of type (T) with at least one of $a$ and $b$ equal to \pm 1 or of type (C) or type (SK). The proof is based on the observation that $\mu\left(p^{r}\right)$ is a positive linear combination of the numbers $A_{a, b}(j)$ defined in (3), where $j$ has the same parity as $r$.

Case 1. $\pi_{F, p}$ is of type (C) or (SK).

In this case $a=p^{\beta} \sigma_{0}$ and $b=\chi(p) \sigma_{0}$, where $0<\beta \leq 1 / 2, \sigma_{0}= \pm 1$ and $|\chi(p)|=1$. Here $\beta=1 / 2$ corresponds to type (SK) and $0<\beta<1 / 2$ corresponds to type (C). Using (3) we get

$$
A_{a, b}(j)=\left(p^{\beta} \sigma_{0}\right)^{j}\left|\sum_{u=0}^{j}\left(p^{\beta} \chi(p)\right)^{-u}\right|^{2} .
$$

Since $\left|p^{\beta} \chi(p)\right| \neq 1$, the sum is never zero. Hence we are in the first case of the lemma if $\sigma_{0}=1$, and in the second case if $\sigma_{0}=-1$.

Case 2. $\pi_{F, p}$ is of type (T).

In this case our hypothesis implies that at least one of $a$ or $b$ equals \pm 1 . Observe that $A_{a, b}(j)$ remains unchanged when we exchange $a$ and $b$. Hence, without loss of generality, we may assume that $a$ is \pm 1 . First assume that $a=1$. Then, from (3) we have

$$
A_{a, b}(j)=\left|\sum_{u=0}^{j} b^{u}\right|^{2} \geq 0 \quad \text { for all } j>0 .
$$

Hence we are in the first case of the lemma. Now assume that $a=-1$. Then, from (3),

$$
A_{a, b}(j)=(-1)^{j}\left|\sum_{u=0}^{j}(-b)^{u}\right|^{2} .
$$

If $b=1$, then we are in the first case of the proposition. If $b=-1$, then we are in the second case of the proposition. Assume that $b \neq \pm 1$. If $r$ is even, then $A_{a, b}(0)=1$ occurs in (2). If $r$ is odd, then $A_{a, b}(1)=-|1-b|^{2}$ occurs in (2). In either case, $\mu\left(p^{r}\right) \neq 0$, so that we are in the second case of the lemma. 
We wish to remark here that we are able to prove the above proposition only by using Proposition 1.1. We cannot carry out the above proof by merely using the unitary classification from [10, [11. Also, note that the hypothesis of the theorem (at least one of $a$ or $b$ is real) forces the local representation to be either the complementary series (type $(\mathrm{C})$ ) or the limits of complementary series $-\beta=1 / 2$ that gives the type (SK) and $\beta=0$ gives the tempered with at least one of $a$ or $b$ equal to \pm 1 .

Lemma 2.1 gives us the following information about the sign of the eigenvalues $\mu\left(p^{r}\right)$ for a fixed prime $p \nmid N$ if we do not make any assumption on the local representation $\pi_{F, p}$ as in Lemma 2.1 .

2.2 Proposition. Let $F \in S_{k}\left(\Gamma_{0}(N)\right)$ be a Hecke eigenform of degree 2 and weight $k>2$. For a fixed prime $p \nmid N$ let $\mu\left(p^{r}\right)$ be the eigenvalue of $F$ for any $r>0$. Then exactly one of the following is true:

1. $\mu\left(p^{r}\right) \geq 0$ for all $r>0$.

2. There are infinitely many $r$ such that $\mu\left(p^{r}\right)>0$ and infinitely many $r$ such that $\mu\left(p^{r}\right)<0$.

Proof. We will distinguish two cases.

Case 1. $\mu\left(p^{r}\right) \geq 0$ for almost all $r$.

In this case we shall prove that $\mu\left(p^{r}\right) \geq 0$ for all $r>0$.

To prove this we will use the following theorem due to Landau on Dirichlet series with non-negative coefficients.

2.3 Theorem (Landau). Suppose $G(s)$ is represented in the half plane $\operatorname{Re}(s)>c$ by the series

$$
G(s)=\sum_{n=1}^{\infty} \frac{a(n)}{n^{s}},
$$

where $c \in \mathbb{R}$ is finite and $a(n) \geq 0$ for all $n \geq n_{0}$. Then either the series in (4) converges for all values of $s$ or the function $G(s)$ has a singularity on the real line at the abscissa of convergence of the series.

Let us return to the proof of Proposition 2.2. Consider the function

$$
G(s)=\frac{1-p^{-1} p^{-2 s}}{\left(1-a p^{-s}\right)\left(1-a^{-1} p^{-s}\right)\left(1-b p^{-s}\right)\left(1-b^{-1} p^{-s}\right)},
$$

where $a=\sigma(p)$ and $b=\sigma(p) \chi_{1}(p)$ as before. Let us set $a\left(p^{r}\right):=\frac{\mu\left(p^{r}\right)}{\left(p^{r}\right)^{k-3 / 2}}$ and $a(n):=0$ if $n$ is not of the form $p^{r}$ for any $r$. From [9], Theorem 4.2, we know that $\left|\mu\left(p^{r}\right)\right| \leq 36\left(p^{r}\right)^{k-1}$, which implies that the series

$$
\sum_{n=1}^{\infty} \frac{a(n)}{n^{s}}=\sum_{r=0}^{\infty} \frac{\mu\left(p^{r}\right)}{\left(p^{r}\right)^{k-\frac{3}{2}}}\left(p^{r}\right)^{-s}
$$

converges absolutely for $\operatorname{Re}(s)>1 / 2$. Hence, from (1), we get

$$
\begin{aligned}
\frac{1-p^{-1} p^{-2 s}}{\left(1-a p^{-s}\right)\left(1-a^{-1} p^{-s}\right)\left(1-b p^{-s}\right)\left(1-b^{-1} p^{-s}\right)}=\sum_{r=0}^{\infty} \frac{\mu\left(p^{r}\right)}{\left(p^{r}\right)^{k-\frac{3}{2}}}\left(p^{r}\right)^{-s} & \\
& \text { for } \operatorname{Re}(s)>\frac{1}{2} .
\end{aligned}
$$


According to the assumption that only finitely many eigenvalues are negative, we see that the above function satisfies the hypothesis of Landau's theorem. Hence either the series converges for all $s$ (in which case $G(s)$ is an entire function) or has a pole on the real line at the abscissa of convergence. Since the function on the left hand side clearly has poles, the first alternative cannot occur, so that there is a pole on the real line. Hence at least one of $a$ or $b$ is real. From Lemma 2.1 we now conclude that $\mu\left(p^{r}\right) \geq 0$ for all $r>0$.

Case 2. $\mu\left(p^{r}\right)<0$ for infinitely many $r$.

In this case we shall prove that there are infinitely many $r$ for which $\mu\left(p^{r}\right)>0$. Assume that there are only finitely many $r$ for which $\mu\left(p^{r}\right)>0$. Similarly as above, we have

$$
\begin{aligned}
-\frac{1-p^{-1} p^{-2 s}}{\left(1-a p^{-s}\right)\left(1-a^{-1} p^{-s}\right)\left(1-b p^{-s}\right)\left(1-b^{-1} p^{-s}\right)}=\sum_{r=0}^{\infty} \frac{-\mu\left(p^{r}\right)}{\left(p^{r}\right)^{k-\frac{3}{2}}}\left(p^{r}\right)^{-s} & \\
& \text { for } \operatorname{Re}(s)>\frac{1}{2} .
\end{aligned}
$$

By our assumption, the Landau argument applies to the series on the right-hand side, proving that at least one of $a$ or $b$ is real. But then Lemma 2.1 implies that $\mu\left(p^{r}\right)$ is positive for infinitely many $r$, a contradiction.

Notice that in Proposition 2.2 we do not get the stronger result as in Lemma 2.1 since a priori it is possible that the local representation $\pi_{F, p}$ is a tempered representation with both $a$ and $b$ not real.

\section{Global Results}

To state the main result of this paper we need to introduce CAP representations and theta lifts. Given an irreducible cuspidal automorphic representation $\pi$ of $\mathrm{GSp}_{4}(\mathbb{A})$ and a proper parabolic subgroup $P=M N$ with levi factor $M$, we say that $\pi$ is CAP (Cuspidal Associated to Parabolic) associated to the parabolic $P$ if there is an irreducible cuspidal automorphic representation $\tau$ of $M$ such that $\pi_{p} \simeq \pi_{p}^{\prime}$ for almost all $p$, where $\pi^{\prime}$ is an irreducible component of $\operatorname{Ind}_{P(\mathbb{A})}^{\mathrm{GSp}_{4}(\mathbb{A})}(\tau)$. Recall that $\mathrm{GSp}_{4}$ has only three parabolic subgroups (up to conjugation), the Siegel parabolic subgroup $P$, the Klingen parabolic $Q$ and the Borel subgroup $B$. We have shown in 9, Corollary 4.5, that if we start with a holomorphic, cuspidal eigenform $F$ of degree 2, weight $k>2$ and level $N$, then any of the corresponding irreducible, cuspidal, automorphic representations $\pi_{F}$ of $\operatorname{GSp}_{4}(\mathbb{A})$ can only be CAP to the Siegel parabolic.

It is known that an irreducible, cuspidal, automorphic representation $\pi$ of $\mathrm{GSp}_{4}(\mathbb{A})$ is CAP to the Siegel parabolic if and only if it is either a theta lift from an irreducible, cuspidal, automorphic representation $\widetilde{\tau}$ of $\widetilde{S L}_{2}(\mathbb{A})$, the metaplectic cover of $\mathrm{SL}_{2}$, or a twist by an idele class character of such a theta lift. It is shown in [8], Theorem 2.2, that $\pi$ is a theta lift if and only if the degree four (spin) $L$ function $L_{\text {Spin }}(s, \pi)$ of $\pi$ has a pole. In [8, Lemma 3.1, it is also shown that the only possible poles of $L_{\operatorname{Spin}}(s, \pi)$ are at $s=3 / 2$ and $s=-1 / 2$. If $\pi$ is a twist of a theta lift by a non-trivial character, then $L_{\mathrm{Spin}}(s, \pi)$ has no poles.

If $\pi_{F}$ is one of the representations corresponding to a holomorphic Siegel cusp form $F$ of weight $k>2$ and level $N$, then $\pi_{F}$ is CAP if and only if either it is a 
theta lift or it is a theta lift twisted by a quadratic character. We get the restriction that the twisting character is quadratic because $\pi_{F}$ has a trivial central character. We have the following information about the local representation $\pi_{F, p}$ for $p \nmid N$ if $\pi_{F}$ is a CAP representation:

1. If $\pi_{F}$ is a theta lift, then for every $p \nmid N$ the local representation $\pi_{F, p}$ is of type $(\mathrm{SK})$ with $\pi_{F, p}$ being the spherical constituent of the induced representation of the form $\nu^{1 / 2} \chi \times \nu^{1 / 2} \chi^{-1} \rtimes \nu^{-1 / 2}$ with $|\chi|=1$.

2. If $\pi_{F}$ is a twist of a theta lift with a quadratic character $\sigma_{0}=\bigotimes \sigma_{0, p}$, then for every $p \nmid N$ for which $\sigma_{0, p}$ is unramified the local representation $\pi_{F, p}$ is of type $(\mathrm{SK})$ with $\pi_{F, p}$ being the spherical constituent of the induced representation of the form $\nu^{1 / 2} \chi \times \nu^{1 / 2} \chi^{-1} \rtimes \nu^{-1 / 2} \sigma_{0, p}$ with $|\chi|=1$.

Now we state our main theorem.

3.1 Theorem. Let $N$ and $k$ be positive integers with $k>2$. Let $F \in S_{k}\left(\Gamma_{0}(N)\right)$ be a Hecke eigenform with Hecke eigenvalues $\mu(n)$ for all $n$ coprime to $N$. Let $\pi_{F}$ be one of the corresponding irreducible, cuspidal, automorphic representations of $\mathrm{GSp}_{4}(\mathbb{A})$. Then the following two statements are equivalent:

1. $\pi_{F}$ is not a theta lift from an irreducible cuspidal automorphic representation of $\widetilde{\mathrm{SL}}_{2}(\mathbb{A})$, the metaplectic cover of $\mathrm{SL}_{2}$.

2. There exists an infinite set $S_{F}$ of prime numbers $p \nmid N$ such that if $p \in S_{F}$, then there are infinitely many $r$ such that $\mu\left(p^{r}\right)>0$ and infinitely many $r$ such that $\mu\left(p^{r}\right)<0$.

Moreover, if $\pi_{F}$ is a theta lift, then we have the stronger statement that $\mu\left(p^{r}\right)>0$ for all $p \nmid N$ and $r>0$.

Proof. First assume that $\pi_{F}$ is a theta lift. Then from the discussion before the statement of the theorem we know that for all $p \nmid N$ the local representation $\pi_{F, p}$ is the spherical constituent of an induced representation of the form $\nu^{1 / 2} \chi \times \nu^{1 / 2} \chi^{-1} \rtimes$ $\nu^{-1 / 2}$ with $|\chi|=1$. Using (3) we get

$$
A_{a, b}(j)=\left(p^{1 / 2}\right)^{j}\left|\sum_{u=0}^{j}\left(p^{1 / 2} \chi(p)\right)^{-u}\right|^{2}>0,
$$

so that $\mu\left(p^{r}\right)>0$ for all $r>0$, as claimed.

Now we will prove the equivalence of the two statements in the theorem. If there exists a prime $p$ such that there are infinitely many $r$ such that $\mu\left(p^{r}\right)>0$ and infinitely many $r$ such that $\mu\left(p^{r}\right)<0$, then from the previous paragraph it is clear that $\pi_{F}$ is not a theta lift. Hence statement 2 implies 1.

Now assume that $\pi_{F}$ is not a theta lift. There are two possibilities :

Case 1. Suppose $\pi_{F}$ is a twist of a theta lift by a non-trivial quadratic character $\sigma_{0}$. Let $S_{F}:=\left\{p \nmid N: \sigma_{0, p}(p)=-1\right\}$. Since $\sigma_{0}$ is a non-trivial character, $S_{F}$ is an infinite set. Now let $p \in S_{F}$. Again using (3) we get

$$
A_{a, b}(j)=\left(-p^{1 / 2}\right)^{j}\left|\sum_{u=0}^{j}\left(p^{1 / 2} \chi(p)\right)^{-u}\right|^{2} .
$$

Thus, by (2), we conclude that $\mu\left(p^{r}\right)>0$ if $r$ is even and $\mu\left(p^{r}\right)<0$ if $r$ is odd. This gives us the desired result.

Case 2. Suppose $\pi_{F}$ is not a CAP representation. 
Let $S$ be the set of primes $p \nmid N$ such that the sequence $\mu\left(p^{r}\right), r>0$, has infinitely many sign changes. Let us assume, contrary to the assertion, that $S$ is finite. First observe that Proposition 2.2 implies that if $p \notin S$, then $\mu\left(p^{r}\right) \geq 0$ for all $r>0$. Set $a(n)=\frac{\mu(n)}{n^{k-3 / 2}}$ if $(n, N)=1$ and $(n, p)=1$ for all $p \in S$ and $a(n)=0$ otherwise. Then $a(n) \geq 0$ for all $n$. We fix an $\epsilon>0$. From [9], Theorem 4.2, there exists a constant $C_{\epsilon}$ such that $|a(n)| \leq C_{\epsilon} n^{1 / 2+\epsilon}$. Let $\tilde{S}:=S \cup\{p$ prime $: p \mid N\}$. From (11) we see that for $\operatorname{Re}(s)$ large enough $(\operatorname{Re}(s)>3 / 2+\epsilon$ will work)

$$
L_{\text {Spin }}^{\tilde{S}}\left(s, \pi_{F}\right)=\zeta^{\tilde{S}}(2 s+1) \sum_{n=1}^{\infty} \frac{a(n)}{n^{s}}
$$

with

$$
\begin{aligned}
L_{\mathrm{Spin}}^{\tilde{S}}\left(s, \pi_{F}\right) & =\prod_{p \notin \tilde{S}} \frac{1}{\left(1-a p^{-s}\right)\left(1-a^{-1} p^{-s}\right)\left(1-b p^{-s}\right)\left(1-b^{-1} p^{-s}\right)} \\
\zeta^{\tilde{S}}(s) & =\prod_{p \notin \tilde{S}} \frac{1}{1-p^{-s}} .
\end{aligned}
$$

Landau's Theorem applies to the series $\sum_{n=1}^{\infty} \frac{a(n)}{n^{s}}$. Hence the abscissa of convergence, say $c_{0}$, of this series is either $-\infty$ (in which case the series represents an entire function) or the series has a pole on the real line at the abscissa of convergence. Now if $c_{0}<0$, then the right-hand side of (17) has a pole at $s=0$ (since $\zeta^{\tilde{S}}(2 s+1)$ has a pole at $s=0$ and $\sum_{n=1}^{\infty} \frac{a(n)}{n^{s}}$ is non-zero at $\left.s=0\right)$. This implies that $L_{\text {Spin }}^{\tilde{S}}\left(s, \pi_{F}\right)$, and hence $L_{\text {Spin }}\left(s, \pi_{F}\right)$, has a pole at $s=0$. But this is not possible, since we have already seen that the spin $L$-function can have a pole only at $s=3 / 2$ or $s=-1 / 2$. Hence $c_{0} \geq 0$. Then Landau's theorem forces $c_{0}=3 / 2$. This implies that $L_{\text {Spin }}\left(s, \pi_{F}\right)$ has a pole at $s=3 / 2$ which forces $\pi_{F}$ to be a theta lift. This contradicts our assumption that $\pi_{F}$ is not a CAP representation, hence proving the theorem.

3.2 Corollary. Let $F \in S_{k}(\operatorname{Sp}(4, \mathbb{Z}))$ be a Hecke eigenform with Hecke eigenvalues $\mu(n)$ for all positive integers $n$. Let $\pi_{F}$ be one of the corresponding irreducible, cuspidal, automorphic representations of $\operatorname{GSp}_{4}(\mathbb{A})$. Then:

1. If $F$ is in the Maaß space, then $\mu(n)>0$ for all $n$.

2. If $F$ is not in the Maaß space, then there exists an infinite set $S_{F}$ of prime numbers $p$ such that if $p \in S_{F}$, then there are infinitely many $r$ such that $\mu\left(p^{r}\right)>0$ and infinitely many $r$ such that $\mu\left(p^{r}\right)<0$.

Proof. By [5] or [7, the eigenform $F$ being in the Maaß space is equivalent to $L_{\text {Spin }}(s, F)$ having poles. By [8], this in turn is equivalent to $\pi_{F}$ being a theta lifting from $\widetilde{\mathrm{SL}}_{2}(\mathbb{A})$. Hence the result is a consequence of Theorem 3.1.

\section{REFERENCES}

[1] Andrianov, A., Zhuravlev, V.: Modular Forms and Hecke Operators. Translations of Mathematical Monographs, vol. 145, AMS, 1995. MR1349824 (96d:11045)

[2] Breulmann, S.: On Hecke eigenforms in the Maaß space. Math. Z. 232, 527-530 (1999). MR:1719682 (2000j:11066)

[3] Cartier, P.: Representations of p-adic groups: A survey. Proc. Symp. Pure Math. 33, vol. 1, 111-155 (1979). MR546593(81e:22029)

[4] Chai, Ch.-L., Faltings, G.: Degeneration of Abelian Varieties. Springer, Ergebnisse d. Math. 22, Berlin, Heidelberg, New York, 1990. MR.1083353 (92d:14036) 
[5] Evdokimov, S. A.: A characterization of the Maass space of Siegel cusp forms of second degree. Math. USSR Sb. 40, 125-133 (1981).

[6] Kohnen, W.: Sign changes of Hecke eigenvalues of Siegel cusp forms of genus two. Proc. Amer. Math. Soc. 135, 997-999 (2007). MR2262899 (2007j:11057)

[7] Oda, T.: On the poles of Andrianov L-functions. Math. Ann. 256, 323-340 (1981). MR626953 (83c:10034)

[8] Piatetski-Shapiro, I.I.: On the Saito-Kurokawa Lifting. Invent. Math. 71, 309-338 (1983). MR689647 (84e:10038)

[9] Pitale, A., Schmidt, R.: Ramanujan type results for Siegel cusp forms of degree 2. Preprint (2007).

[10] Rodier, F.: Sur les représentations non ramifiées des groupes réductifs $p$-adiques; l'exemple de GSp(4). Bull. Soc. Math. France 116, 15-42 (1988). MR946277 (89i:22033)

[11] Sally, P., Tadić, M.: Induced rerpesentations and classifications for $\operatorname{GSp}(2, F)$ and $\operatorname{SSp}(2, F)$. Bull. Soc. Math. France 121, Mem. 52, 75-133 (1993). MR.1212952 (94e:22030)

Department of Mathematics, University of Oklahoma, Norman, Oklahoma 73019

E-mail address: ameya@math.ou.edu

Department of Mathematics, University of Oklahoma, Norman, Oklahoma 73019

E-mail address: rschmidt@math.ou.edu 